Jorge Mesquita Huet Machado ${ }^{1}$

Vilma Sousa Santana ${ }^{2}$

Augusto Campos ${ }^{3}$

Silvia Ferrite ${ }^{4}$

Maria Claudia Peres ${ }^{2}$

Adriana Galdino ${ }^{5}$

Renata Vasconcelos Neto ${ }^{6}$

Roque Manoel Perusso da Veiga ${ }^{6}$

Maria Cláudia Lisboa ${ }^{2}$

Ana Paula Lopes dos Santos

${ }^{1}$ Coordenação de Programas e Projetos, Diretoria Regional de Brasília, Fundação Oswaldo Cruz. Brasília, DF, Brasil.

2 Programa Integrado em Saúde Ambiental e do Trabalhador, Instituto de Saúde Coletiva, Universidade Federal da Bahia. Salvador, BA, Brasil.

${ }^{3}$ Escola de Governo, Diretoria Regional de Brasília, Fundação Oswaldo Cruz. Brasília, DF, Brasil.

${ }^{4}$ Departamento de Fonoaudiologia, Instituto de Ciências da Saúde, Universidade Federal da Bahia. Salvador, BA, Brasil. ${ }^{5}$ Departamento de Saúde, Universidade Estadual do Sudoeste da Bahia. Jequié, BA, Brasil.

${ }^{6}$ Coordenação Geral da Saúde do Trabalhador, Secretaria da Vigilância em Saúde, Ministério da Saúde. Brasília, DF, Brasil.

${ }^{7}$ Departamento de Psicologia, Universidade Federal Fluminense. Niterói, RJ, Brasil.

Contato:

Jorge Mesquita Huet Machado

E-mail:

jorgemesquita@yahoo.com.br

Financiamento: Ministério da Saúde. Portaria GM/MS no 1679/02.

Trabalho baseado no $1^{\circ}$ Inventário de Saúde do Trabalhador 2009 - Avaliação da Rede Nacional de Atenção Integral em Saúde do Trabalhador, 2008-2009, desenvolvido pelos autores junto à Coordenação Geral de Saúde do TrabaIhador da Secretaria de Vigilância em Saúde do Ministério da Saúde.

Os autores declaram não haver conflitos de interesse.

Recebido: 29/03/2012

Revisado: 03/07/2012

Aprovado: 10/01/2013

\section{Situação da Rede Nacional de Atenção Integral em Saúde do Trabalhador (Renast) no Brasil, 2008-2009}

\author{
Status of the Brazilian National Workers' Health Network, \\ 2008-2009
}

\section{Resumo}

Este artigo apresenta um recorte da pesquisa " 1 을 Inventário de Saúde do Trabalhador 2009”, desenvolvida pela Coordenação Geral de Saúde do Trabalhador do Ministério da Saúde. Buscou-se identificar lacunas e potencialidades da implantação da Rede Nacional de Atenção Integral em Saúde do Trabalhador (Renast), focando as ações de Vigilância em Saúde do Trabalhador (Visat) e variáveis estruturantes dos Centros de Referência em Saúde do Trabalhador (Cerest), como o financiamento, a equipe profissional e a participação dos trabalhadores. Analisaram-se as atividades de Visat e destacaram-se as articulações dos Cerests intra e intersetoriais, entre elas instâncias regionais da Previdência Social e universidades, além das internas ao SUS, sobretudo com instâncias da Vigilância em Saúde. Os resultados demonstraram que as ações de Visat estão acontecendo na maioria dos Cerests $(58,4 \%)$ e que há uma adequação estrutural e da equipe, além de relativa participação dos trabalhadores nas Comissões Intersetoriais de Saúde do Trabalhador (CIST) dos Conselhos de Saúde. São apresentadas considerações para a construção de parâmetros para futuras pesquisas e recomendações visando ao fortalecimento da Renast. Destaca-se a necessidade de intensificação da capacidade de análise das informações decorrentes das ações de vigilância e do registro de casos.

Palavras-chave: vigilância em saúde do trabalhador; avaliação em saúde; redes de serviços de saúde.

\section{Abstract}

This paper presents partial records of the first report on worker's health 2009 produced by the Workers' Health General Coordination (CGSAT) of the Ministry of Health. It identifies gaps and potentialities in implanting the Brazilian National Network for Worker's Health Integrated Care (Renast). It is focused on actions performed by the Worker's Health Surveillance (VISAT) and structural indexes of the Workers' Health Reference Centers (Cerest), such as its financial flow, its professional team and the workers' participation. It also presents the VISAT activities carried out and the inter and intra sector articulations of the Cerests, among then, universities as well as the regional spheres of the Brazilian Social Security Ministry. The results evidences that VISAT actions are taking place in most Cerests $(58,4 \%)$, and show that the services physical structure and technical teams are appropriate and also that there is a reasonable participation of workers in the Workers' Heath Inter-sector Commissions (CIST) of the Regional Health Committees. The paper presents parameters viewing future research and recommendations aimed at empowering Renast. Finally it emphasizes the need to intensify the capacity to analyze information resulting from surveillance actions and case records.

Keywords: worker's health surveillance; health assessment; health services network. 


\section{Introdução}

O presente trabalho apresenta um panorama de implantação da Rede Nacional de Atenção Integral em Saúde do Trabalhador (Renast) no Sistema Unico de Saúde (SUS) para o ano base de 2009. Para tal, são selecionadas algumas questões presentes no Inventário da Coordenação Geral de Saúde do Trabalhador (CGSAT), da Secretaria de Vigilância em Saúde (SVS), do Ministério da Saúde de 2008-2009 (BRASIL, 2011). A pesquisa aqui relatada representa um recorte dessa etapa inicial do processo de monitoramento sistemático dos Centros de Referência em Saúde do Trabalhador (Cerest), compartilhado pela CGSAT-SVS em parceria com o Centro Colaborador em Vigilância dos Acidentes de Trabalho, da Universidade Federal da Bahia (CC-UFBA) e com o Programa de Promoção de Saúde Ambiente e Trabalho, da Fiocruz-Brasília. Tal monitoramento tem como objetivo a identificação das lacunas e das potencialidades dos Cerests, visando à redefinição dos rumos e das estratégias de apoio para o fortalecimento da Renast.

A Renast se constitui em uma rede de Cerests, que se concretiza com ações integradas transversais em Saúde do Trabalhador, que incluem a gestão, a informação, a definição e o compartilhamento das ações de vigilância e de assistência em todos os níveis de atenção. Nesse contexto, os Cerests são compreendidos como polos irradiadores estratégicos na ação de matriciamento da Renast no SUS.

A importância e a necessidade de monitoramento do processo de estruturação da Renast e da avaliação das suas ações, em especial as desenvolvidas pelos Cerests, já foi objeto de manifestações de parte de diversos autores, assim como em depoimentos narrados em encontros e seminários da Renast. Para Dias e Hoefel (2005), a Renast se constituiu em uma estratégia bem-sucedida, embora perpassada por dificuldades, como a diferença da área regional de cobertura dos Cerests em relação à organização de base municipal adotada pelo SUS. Relataram ainda a frágil articulação intrassetorial, a falta de clareza na definição das instâncias de coordenação das ações e dos direcionamentos para a pactuação entre os diferentes níveis de governo no planejamento estadual, regional ou municipal. Estes limites vêm sendo discutidos, mas ainda requerem um maior detalhamento das suas causas, de modo a subsidiar sua superação, estabelecendo avanços e inovações.

Em uma análise da incorporação da Saúde do Trabalhador nos 20 anos do SUS, Santana e Silva (2009) verificaram que os Cerests e as ações de saúde do trabalhador haviam se expandido para todas as unidades da federação, alcançando-se uma razão Cerest: população de trabalhadores aproximadamente homogênea no país. Argumentava-se, no entanto, que esse dado não significava igualdade, existindo diferenças na qualidade, no acesso e na oferta de serviços, sugerindo também uma avaliação específica da implantação, o que também está previsto no respectivo Manual de Gestão e Gerenciamento (BRASIL, 2006).

Em estudo qualitativo sobre os limites e os avanços das ações de ST no SUS, em Minas Gerais, Dias et al. (2010) concluíram que os Cerests representam um avanço na institucionalização das ações de saúde do trabalhador, embora faltem claras definições dos critérios de regionalização, das estruturas componentes da Renast, do papel dos Cerests, dentre outros aspectos.

Santos (2010), ao pesquisar experiências consideradas exitosas desenvolvidas pelos Cerests de São Paulo/SP, conclui que a potencialidade delas está na formação de redes intra e interinstitucionais em torno de projetos de ação definidos como prioritários, assim como na apresentação de possibilidades de transcender a abordagem do processo saúde e doença, pautada tradicionalmente por ações médico-assistenciais individuais, na perspectiva de ações que visam à prevenção, à promoção e à vigilância em saúde do trabalhador.

\section{Mateial e métodos}

Este estudo descritivo apresenta um panorama da implantação dos Cerests no âmbito nacional, cobrindo todas as unidades que prestaram informações a CGAST/Ministério da Saúde no período do levantamento, que configurou o " 1 을 Inventário de Saúde do Trabalhador, 2009: Avaliação da Rede Nacional de Atenção Integral em Saúde do Trabalhador, 2008-2009" (BRASIL, 2011). Neste artigo, foram selecionadas questões referentes ao financiamento, à equipe profissional, à participação social e ao desempenho das atividades de vigilância em saúde do trabalhador.

A metodologia adotada na avaliação do inventário foi desenvolvida a partir do levantamento realizado por Lacaz, Machado e Porto (2002) em conjunto com técnicos da Fiocruz, da CGSAT e do CC-UFBA. Contou com crítica e sugestões do GT de Vigilância em Saúde do Trabalhador, composto por técnicos da CGSAT, colaboradores de grupos acadêmicos e gestores estaduais da Renast, e com recomendações de oficina específica também organizada por esses atores institucionais. Destaca-se que as atividades de preparação do inventário ocorreram simultaneamente ao estudo do CC-UFBA-CGSAT, que focalizava especificamente a avaliação das ações da vigilância dos acidentes de trabalho na Renast (GALDINO, SANTANA; FERRITE, 2012).

Essencialmente, o método adotado organiza o acompanhamento das ações dos Cerests em três di- 
mensões constituídas por blocos de variáveis. São elas: Questionário 1) de estrutura, em que as equipes, o controle social e o financiamento estão enquadrados; Questionário 2) o bloco de ações, em que as ações de vigilância estão incluídas, e; 3) de impacto, representado pelos registros de casos e perfil epidemiológico, dimensão não incluída nos questionários aplicados, mas disponível no Sistema Nacional de Agravos Notificáveis (SINAN-NET) do SUS.

Os dados do inventário (BRASIL, 2011) foram coletados utilizando-se a ferramenta FormSUS, do Departamento de Informática do SUS (DATASUS). Este é um sistema padronizado segundo normas adotadas pelo Ministério da Saúde para obtenção de dados em-linha. Neste sistema, dados são coletados com formulários virtuais pela internet e armazenados em bases eletrônicas. A coleta se iniciou em maio de 2009, ocasião em que foi enviada a todos os Cerests (140) uma correspondência eletrônica informando sobre os objetivos da avaliação, convidando-os a fornecerem dados nos questionários eletrônicos do FormSus especificados. Em setembro de 2009 foi encerrada a coleta de dados. As informações de financiamento foram coletadas em documentos e relatórios dos arquivos da CGSAT. Os dados do inventário se referem ao ano de 2008 e ao primeiro semestre de 2009. Foram recebidas 137 respostas referentes ao Questionário 1, sobre os aspectos estruturais e da capacidade instalada, e 128 respostas referentes ao Questionário 2, sobre aspectos relacionados à vigilância em saúde do trabalhador.

A fim de se proceder à análise dos resultados dos dados coletados, em primeiro lugar foi elaborado um diagrama descrevendo as atividades e os fluxos envolvidos nas ações da Renast; em seguida, um mo- delo lógico correspondente, definindo-se construtos teóricos, suas respectivas dimensões (estrutura, processos, impactos) componentes (variáveis relativas a distintas dimensões) e interrelações. Parte desse modelo foi apresentada e discutida em uma das reuniões do Grupo de Trabalho da Vigilância em Saúde do Trabalhador, composto por técnicos da CGSAT, colaboradores de grupos acadêmicos e gestores estaduais da Renast, em maio de 2009, em Brasília.

No que se refere a aspectos éticos, o presente estudo foi realizado a partir de dados secundários publicados pelo Ministério da Saúde (BRASIL, 2011), envolvendo informações públicas.

\section{Resultados}

\section{Financiamento}

Os valores relativos ao repasse financeiro do $\mathrm{Mi}-$ nistério da Saúde para os Cerest, entre 2003 a 2009, são mostrados na Figura 1. Nota-se uma tendência crescente linear dos valores, refletindo tanto a consolidação do fluxo de recursos fundo a fundo, quanto a própria execução do programa de expansão dos Cerests (BRASIL, 2009).

Na Tabela 1, mostram-se as distribuições dos gastos segundo ações específicas dos Cerests. Nota-se que, no país, as maiores proporções ocorreram para custeio operacional $(22,8 \%)$ e capacitação externa (19,6\%). De acordo com a instância administrativa, as ações operacionais contaram com 20,6\% no nível estadual e $22,8 \%$ no regional, e as ações de capacitação externa, com a maior concentração isolada no nível estadual (29,9\%).

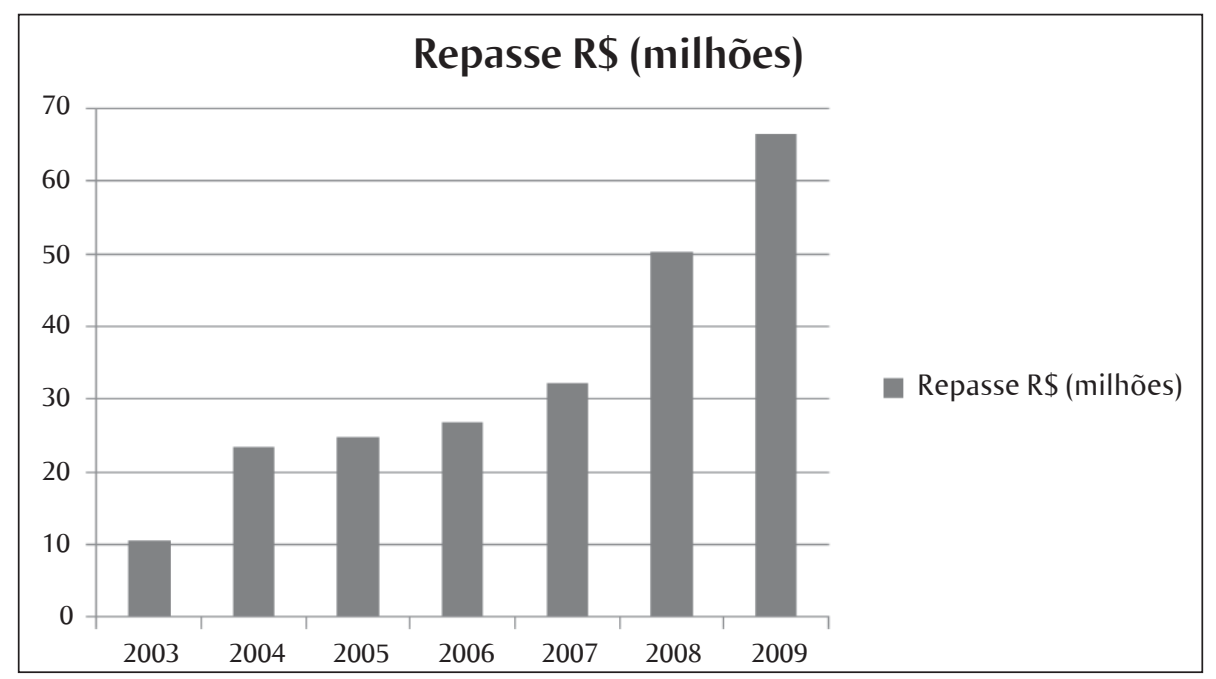

Fonte: $1^{\circ}$ Inventário de Saúde do Trabalhador: Avaliação da Rede Nacional de Atenção Integral em Saúde do Trabalhador, 2008-2009 (BRASIL, 2010, p. 21)

Figura 1 Total de repasse de recursos financeiros, em milhões de reais ( $R \$$ ), para os Cerest, no período de 2003-2009. Brasil, 2009 
Tabela 1 Proporção de gastos segundo finalidades específicas por tipo de abrangência dos Cerest, 2008-2009

\begin{tabular}{lccc}
\hline \multirow{2}{*}{ Especificação } & $\begin{array}{c}\text { Estadual }(n=20) \\
(\%)\end{array}$ & $\begin{array}{c}\text { Regional }(n=54) \\
(\%)\end{array}$ & $\begin{array}{c}\text { Brasil }(n=74) \\
(\%)\end{array}$ \\
\hline Ações de capacitação & 29,9 & 15,8 & 19,6 \\
Controle social & 7,5 & 6,2 & 6,5 \\
Custeio operacional & 20,6 & 23,7 & 22,8 \\
Pagamento de pessoal & 5,5 & 12,5 & 10,6 \\
Capacitação do Cerest & 13,5 & 9,2 & 10,4 \\
Ações de vigilância & 8,8 & 9,6 & 9,4 \\
Ações assistenciais & 10,3 & 6,7 & 7,7 \\
Outros & 4,1 & 16,3 & 13,0 \\
\hline
\end{tabular}

Fonte: $1^{\circ}$ Inventário de Saúde do Trabalhador: Avaliação da Rede Nacional de Atenção Integral em Saúde do Trabalhador, 2008-2009 (BRASIL, 2010, p. 22)

Esses recursos deveriam contar com contrapartida municipal ou estadual e, de fato, apenas $23,9 \%$ dos Cerests utilizavam exclusivamente recursos federais à época do estudo, evidenciando a participação positiva das esferas estaduais e municipais no compartilhamento do aporte financeiro aos Cerests. Esta proporção é maior nos Cerests estaduais $(30,8 \%)$ em comparação com os regionais $(22,4 \%)$. Verifica-se que os estaduais de Rondônia e Roraima (região Norte) não recebiam, à época, contrapartida financeira local ou estadual, bem como os de Alagoas, Paraíba e Rio Grande do Norte (região Nordeste), Espírito Santo, Santa Catarina e Mato Grosso, nas demais regiões do país.

Estes resultados são consonantes com narrativas e relatórios de seminários de avaliações de gestores da Renast. Além disso, nesses encontros ficam evidentes problemas que vão desde a lógica da alocação orçamentária até a execução financeira e seu monitoramento. Por exemplo, os repasses de recursos para os Cerests obedecem a um único critério de valor, mensal, igual para todos eles. Isso pode produzir iniquidades devido às enormes diferenças de extensão de áreas de cobertura, do número de trabalhadores alvo de cada Cerest, além da dimensão e gravidade das necessidades relativas às condições de trabalho e saúde do trabalhador. Ou seja, não são considerados os indicadores epidemiológicos ou demográficos, bem como prioridades definidas a partir de uma análise de situação de saúde, a exemplo de outras ações específicas desenvolvidas pelo próprio SUS. Outros problemas decorrem da grande burocracia na gestão dos recursos, enfrentada pelos gestores que trabalham "na ponta" e lidam diretamente com as demandas operacionais em nível local. São muitas as queixas de falta de recursos para as atividades específicas de ST, embora os repasses da Renast se acumulem, conforme analisado por Machado e Santos (2011).

Há relatos de grandes somas de recursos não utilizados que não chegam ao nível operacional da gestão. Outras situações semelhantes relacionam-se a uma intenção estratégica para o uso em despesas de maior porte, como a aquisição de veículos ou adaptações das instalações físicas. Em outros, ao contrário, há falta de recursos devido ao crônico subfinanciamento da saúde nos orçamentos das prefeituras. Não se considerou aqui a análise da qualidade do uso do recurso, o que deve ser foco de estudos específicos. Vale notar, no entanto, que algumas inconsistências nas respostas indicam a necessidade de melhorar, nos próximos inventários, a elaboração das perguntas relativas ao financiamento e aos gastos, de modo a facilitar a sua compreensão pelos respondentes.

Os resultados demonstram um equilíbrio entre os gastos com Vigilância e Assistência e também uma predominância de gastos com formação, o que pode ser considerado coerente com o estágio inicial de implantação da Renast, em que há uma transição do Cerest como serviço de execução, inclusive de ações assistenciais, para um modelo de Cerest que seja mais organizador de ações com uma ênfase nas ações de vigilância.

\section{Equipe profissional}

A Tabela 2 aponta que a maioria dos Cerests possui pessoal compatível $(71,5 \%)$ com os parâmetros definidos como equipe mínima, segundo os critérios propostos pelo Manual da Renast (BRASIL, 2006). Contar com a equipe mínima prevista foi mais comum entre os Cerests estaduais $(80,0 \%)$ do que entre os regionais $(69,5 \%)$. No entanto, contar com uma equipe mínima de profissionais de saúde não significa, necessariamente, que esta equipe seja adequada às demandas ou às necessidades da população coberta. Nota-se que, na discussão do Manual da Renast, a adequação da equipe mínima é rediscutida à luz de uma maior flexibilidade no perfil dos profissionais que devem ser adequados a uma ação de acompanhamento regional.

Um aspecto preocupante foi a avaliação da adequação das equipes frente às demandas dos Cerest. 
Tabela 2 Distribuição das respostas dos Cerests sobre sua estrutura de pessoal, de acordo com as subdimensões. Brasil, 2009

\begin{tabular}{|c|c|c|c|c|c|c|}
\hline \multirow{2}{*}{ Subdimensões/variáveis } & \multicolumn{2}{|c|}{$\begin{array}{c}\text { Cerest } \\
\text { Estaduais }\end{array}$} & \multicolumn{2}{|c|}{$\begin{array}{c}\text { Cerest } \\
\text { Regionais }\end{array}$} & \multicolumn{2}{|c|}{ Total } \\
\hline & № & $\%$ & № & $\%$ & $N^{\circ}$ & $\%$ \\
\hline \multicolumn{7}{|l|}{ Cerest com equipe mínima* } \\
\hline Sim & 20 & 80,0 & 73 & 69,5 & 93 & 71,5 \\
\hline Total de Cerests respondentes & 25 & 100,0 & 105 & 100,0 & 125 & 100,0 \\
\hline \multicolumn{7}{|l|}{$\begin{array}{l}\text { Avaliação da adequação da equipe em } \\
\text { relação à demanda }\end{array}$} \\
\hline Péssima & 0 & - & 1 & 1,0 & 1 & 0,8 \\
\hline Ruim & 3 & 2,5 & 7 & 6,7 & 10 & 7,8 \\
\hline Regular & 9 & 37,5 & 35 & 33,3 & 44 & 34,1 \\
\hline Boa & 12 & 50,0 & 56 & 53,3 & 68 & 52,7 \\
\hline Excelente & 0 & - & 6 & 5,7 & 6 & 4,7 \\
\hline Total de Cerests respondentes & 24 & 100,0 & 105 & 100,0 & 129 & 100,0 \\
\hline \multicolumn{7}{|l|}{ Vínculo de trabalho } \\
\hline Servidor & 99 & 83,9 & 664 & 72,6 & 763 & 73,9 \\
\hline Terceirizado celetista & 15 & 12,7 & 80 & 8,7 & 95 & 9,2 \\
\hline Prestação de serviços & 0 & - & 40 & 4,4 & 40 & 3,9 \\
\hline Estagiários/estudantes & 0 & - & 21 & 2,3 & 21 & 2,0 \\
\hline Outros & 4 & 3,4 & 110 & 12,0 & 114 & 11,0 \\
\hline Total de trabalhadores dos Cerests*** & 118 & 100,0 & 915 & 100,0 & 1033 & 100,0 \\
\hline
\end{tabular}

Embora a maior proporção considere boa $(50,0 \%$ nos estaduais) e boa e excelente $(59,0 \%$ nos regionais), uma considerável proporção está na faixa de adequação regular (37,5\% nos estaduais e $33,3 \%$ nos regionais). Três Cerests estaduais e sete regionais consideraram "ruim" a capacitação/desempenho de suas respectivas equipes. Apenas um Cerest regional considerou sua equipe como péssima em relação às suas necessidades (Tabela 2).

Em relação à composição das equipes, verifica-se que a maior parte era composta por servidores públicos estatuários $(73,9 \%)$, ao contrário do que é visto na atenção básica (FACCHINI et al., 2006) e no nível nacional do SUS, com maior proporção de celetistas ou de vínculos de emprego precários. Nos Cerest estaduais, a proporção de servidores é $83,9 \%$, maior que nos regionais $(72,6 \%)$ (Tabela 2), o que revela, relativamente, maiores dificuldades em nível municipal para a contratação de servidores.
Em resumo, a situação relativa ao vínculo de trabalho nos Cerests é aparentemente boa, considerando o cenário de 2008. Essa situação contrasta com a observada em outros programas estratégicos do SUS, como o da Saúde da Família (FACCHINI et al., 2006). Deve-se apontar, no entanto, que é frequentemente relatada a existência de focos de vínculos precários de emprego, com alta rotatividade de técnicos, especial dos médicos, nos Cerest (SANTOS, 2010).

Dados sobre a qualificação e a formação das equipes não foram registrados com o detalhamento necessário que permitisse completa avaliação. Entretanto, com os relatórios e as narrativas já mencionados, pode-se inferir que é grande a demanda por formação especializada em nível de pós-graduação, sob a forma de Mestrado Profissional e/ou Strictu Sensu, como Mestrado e Doutorado. A formação em ST no país precisa de estudos amplos e aprofundados sobre necessidades, demandas, perfis de formação, habilidades e competências, bem como discussões em torno 
do formato pedagógico (RAMOS, 2008; SANTANA; SILVA, 2009). Experiências demonstram que o ensino de profissionais em ST é um desafio e que o formato tradicional de aulas expositivas e exercícios práticos em classe não parece ser muito eficiente ou mobilizador. Este desafio deve ser enfrentado a partir do engajamento de professores e profissionais dos serviços, em conjunto com especialistas em educação de profissionais, possivelmente com maior participação de atividades práticas em serviço, alunos-equipe, com uma formação voltada para a solução de problemas cotidianos. A grande necessidade de oportunidades de formação, a concentração de pessoal qualificado em algumas regiões, dentre outros aspectos, impõem a adoção de modalidades como a de educação a distância, com a garantia de momentos presenciais e tutoria qualificada. Estas transformações requerem grandes investimentos na preparação de recursos instrucionais. De uma forma geral, um esforço concentrado deve ser dirigido à formação de profissionais de ST em todos os níveis (SANTOS, 2010).

\section{Participação dos trabalhadores e controle social}

Os dados revelaram que a participação do controle social na gestão da Renast é ainda limitada. Conselhos Gestores (CG) estão implantados e em funcionamento em apenas dois $(8,0 \%)$ dos Cerests estaduais (Bahia e Ceará) e em aproximadamente metade dos regionais. Existem Comissões Intersetoriais de Saúde do Trabalhador (Cist) na maioria dos Cerests estaduais $(\mathrm{n}=19 ; 76,0 \%)$, enquanto que as Cists municipais foram instaladas em apenas $9(36,0 \%)$ dos municípios sede dos Cerests regionais. Na região Sul, todos os Cerests estaduais e regionais possuíam Cists estadual. Um aspecto positivo é que a maioria $(80,3 \%)$ referiu envolver representantes de trabalhadores nas suas programações anuais (Tabela 3). Na região Sul, novamente a situação foi mais favorável, com trabalhadores envolvidos em todos os Cerests estaduais. Nos Cerests regionais da região Norte, isso apenas ocorreu nos estados do Pará e Tocantins.

A composição das Cists, descrita na Tabela 3, mostra uma forte presença dos sindicatos de trabalhadores e de conselheiros de saúde, 93,9\% e 87,8\%, respectivamente. Essa tendência é maior nas Cist descritas pelos Cerests regionais, em que predominam as Cists municipais. As diferenças na proporção dos componentes descritos pelos Cerests estaduais e regionais decorrem das diferenças de estruturação do controle social nos âmbitos estadual e municipal. Na composição das Cists estaduais, há também uma forte proporção de participação das centrais sindicais $(85,0 \%)$ e das universidades $(65,0 \%)$, o que não ocorre nas Cists municipais, que contam com essas representações em apenas $45,2 \%$ e $38,7 \%$, respectivamente. A presença de representantes da Previdência Social e do Ministério do Trabalho e Emprego ocorre em $56,1 \%$ e $31,7 \%$, respectivamente, sendo mais forte a presença dessas instituições em âmbito estadual. Isto demonstra certa dificuldade da presença dessas instituições em nível local, nos municípios, o que ocorre também com as universidades.

Deve-se destacar a presença do Ministério Público (MP) na composição das Cists, embora referida apenas para $20,0 \%$ entre estaduais e $17,7 \%$, entre municipais (Tabela 3). A interação com essa instituição tem viabilizado ações intersetoriais necessárias para a solução de problemas da saúde do trabalhador que ultrapassam os limites setoriais da saúde, como na erradicação do trabalho infantil. A presença sistemática do MP em fóruns da saúde vem permitindo, cada vez mais, melhor direcionalidade de suas ações para as prioridades do SUS.

Por outro lado, a representação patronal no âmbito da Renast é alvo de controvérsias. Há duas tendências de pensamento a respeito da sua incorporação: uma depende da função que as Cists exercem de fato, ou seja, se é um fórum de negociação e discussão de processos de implantação por melhores condições de trabalho, na perspectiva da promoção da saúde dos trabalhadores; outra é se representam, como prescrito formalmente, uma instância de controle social vinculada à ação do SUS, na qual o planejamento e a avaliação de suas ações é atividade fundamental e predominante. No primeiro caso a presença patronal seria bem-vinda, pois qualquer negociação de condições de trabalho requer a representação do patronato; já no segundo, há de se considerar que as representações dos empregadores tendem a concentrar poder e a exercê-lo em detrimento dos direitos dos trabalhadores, relativizando os problemas das condições de trabalho e das situações de risco para a saúde, distantes de um alinhamento lógico com as diretrizes sanitárias.

A presença de representantes da Vigilância Sanitária, Epidemiológica e Ambiental na composição das Cist, especialmente nas municipais, indica uma possível articulação intrassetorial, pelo menos potencial. A participação de trabalhadores e movimentos sociais no debate de propostas e programas propicia uma melhor integração entre as demandas e as necessidades, assim como entre instituições públicas e instâncias executivas, permitindo maior eficiência nas repostas dos serviços e, por conseguinte, melhoria das condições de saúde dos trabalhadores. Todavia, há de se considerar que a presença de representantes de trabalhadores em órgãos gestores não garante representatividade, nem participação política efetiva de grupos sociais invisíveis ou de pequeno capital político nas decisões. O primeiro a se considerar é que muitas instâncias colegiadas de decisão se transformam ou operam como espaços de litígios corporativistas, pendendo para um papel apenas burocrático e cartorial. Mesmo quando refletem discussões relevantes e trazem contribuições positivas para o aprimoramento das políticas e das ações, podem não dar voz e expressão política às ne- 
cessidades de grupos vulneráveis ou pouco organizados, como os segmentos dos trabalhadores informais ou trabalhadores, cuja própria existência é ilegal, como as crianças trabalhadoras e trabalhadores em situações análogas ao da escravidão. Portanto, é fundamental a incorporação de atores que possam dar voz a grupos de pequeno capital político ou estejam antenados com os grandes problemas da saúde dos trabalhadores, não necessariamente os captados pelos sistemas clássicos de produção de informação em Saúde do Trabalhador. Exemplo disso são as Organizações não Governamentais (ONG), redes internacionais e nacionais, o MP, instituições acadêmicas e grupos de estudos e pesquisas, nacionais ou internacionais, bem como a mídia.

A participação dos trabalhadores nas ações de programação anual foi referida em $80,3 \%$ dos Cerests, sendo mais forte nos regionais $(83,5 \%)$ e relativamente menor nos estaduais $(66,7 \%)$ segundo a Tabela 3. Esta situação contrasta com a participação de representantes dos trabalhadores nas reuniões de planejamento e avaliação das ações dos Cerests, referida por apenas $32,0 \%$ dos estaduais e $56,0 \%$ dos regionais como "frequentemente" ou "sempre" (Tabela 3). A baixa frequência da participação de trabalhadores nas ações de Vigilância em Saúde do Trabalhador ficou demonstrada no fato de que apenas $24,0 \%$ dos Cerest referiram uma participação "frequente" $(20,0 \%)$, e "sempre" (4,0\%) (Tabela 3). A situação é ainda mais inadequada nos Cerests regionais, o que demanda atenção para melhor compreensão.

Por um lado, há dificuldades práticas de envolvimento dos trabalhadores relativas à compatibilidade com as suas tarefas nas inspeções sanitária dos ambientes de trabalho. No Brasil, o grau de instabilidade das relações de trabalho gera situações em que os trabalhadores, muitas vezes, sentem-se constrangidos em participar de inspeções, pois, ao revelarem problemas gerenciais e operacionais que possam resultar em punições dos empregadores, poderiam ser alvo de represálias, até mesmo por parte de colegas de trabalho. Por outro lado, é amplamente reconhecida a importância da participação dos trabalhadores em todas as atividades envolvidas com a ST, não apenas para conhecimento mais pertinente da realidade, mas também para garantir a efetividade das intervenções em seus diferentes aspectos, sejam educacionais, de cumprimento de normas e de pressão junto aos empregadores para a proteção da sua saúde e segurança. A pequena participação de trabalhadores pode expressar uma valorização excessiva de aspectos normativos, prescritivos e técnicos em detrimento do conhecimento do trabalhador sobre o trabalho por ele desenvolvido. A ausência de trabalhadores nas ações de Visat não atende às orientações de organismos internacionais como a Organização Internacional do Trabalho (OIT) e a Organização Mundial da Saúde (OMS), nem pressupostos teóricos e institucionais de referência (MACHADO, 1996; BRASIL, 1998; BRASIL, 2012).
O saber do trabalhador é fundamental para a compreensão e a validação do conhecimento técnico e, embora não seja a única contribuição na construção de ambientes seguros e saudáveis, é a mais negligenciada. Notar que alguns riscos químicos, por exemplo, são inodoros e que exposições podem cursar por longos períodos sem manifestação de intoxicação aguda ou efeitos crônicos são de difícil reconhecimento por parte dos próprios trabalhadores. É necessário que eles sejam informados dos avanços do conhecimento científico e que contribuam com a sua tradução em políticas e programas, com a sua experiência e intuição. A sua participação nas ações da Visat precisa ser ampliada e qualificada, isto é, protegida de retaliações, valorizada por técnicos, empregadores e pelos próprios trabalhadores.

O conhecimento acerca dos efeitos sobre a saúde é resultado da combinação de diferentes saberes. Contribui para isso o conhecimento científico de várias origens como da Toxicologia, da Genética, das Ciências Sociais, da Ergonomia, da Epidemiologia, da Medicina, dentre outras. A identificação de riscos e situações críticas, discussões e negociações de alternativas tecnológicas mais adequadas à saúde dos trabalhadores, a articulação de parcerias políticas e estratégicas para a construção de mecanismos de proteção social dos atores envolvidos são esperadas e devem ser reforçadas.

\section{Ações desenvolvidas pelos Cerests}

A dimensão das ações desenvolvidas expressa a capacidade de resposta dos Cerests às demandas e às necessidades da região de cobertura, bem como de resposta às diretivas da Política Nacional de Saúde do Trabalhador, dos manuais da Renast e do Cerest. Neste inventário, o foco foi nas ações de Visat por seu caráter estruturante das ações de Saúde do Trabalhador na rede do SUS.

Reiterando que esses dados se referem a 20082009, observou-se que a maioria dos Cerests referiu realizar inspeções para avaliação de ambientes de trabalho $(58,4 \%)$ (Tabela 4). Considerando-se as respostas para "em funcionamento pleno" e "em funcionamento", foram 16,8\% e 41,6\%, respectivamente. Uma parcela dos Cerests (19,2\%) referiu estar em fase de implantação e apenas 4,0\% referiram não haver previsão no planejamento dessas atividades (Tabela 4). Na região Sul, todos os Cerests estaduais realizavam inspeções em ambientes de trabalho, enquanto na região Norte apenas os estados do Amapá e Tocantins. Poucos Cerests estaduais referiram avaliar as ações de Visat $(n=5)$, sendo que nenhum desses se localizava nas regiões Norte e Centro-Oeste (Tabela 4). Entre os Cerests regionais, também foi pouco comum a realização dessas avaliações, verificando-se que, na região Norte, apenas os dois $(\mathrm{n}=2)$ do Tocantins e, na região Centro-Oeste, um $(\mathrm{n}=1)$ em Goiás referiram "funcionamento" ou "pleno funcionamento". 
Tabela 3 Situação da gestão participativa, do controle social e da participação dos trabalhadores. Brasil, 2009

\begin{tabular}{|c|c|c|c|c|c|c|}
\hline \multirow{2}{*}{ Variáveis* } & \multicolumn{2}{|c|}{ Cerests estaduais } & \multicolumn{2}{|c|}{ Cerests regionais } & \multicolumn{2}{|c|}{ Total } \\
\hline & № **⿻丷木 $^{2}$ & $\%$ & № & $\%$ & $N^{\circ}$ & $\%$ \\
\hline Tem conselho gestor $(n=129)$ & 2 & 8,0 & 52 & 50,0 & 54 & 41,9 \\
\hline Tem Cist****** $(n=143)$ & 20 & 76,9 & 62 & 60,8 & 82 & 57,3 \\
\hline Composição da Cist $(n=82)$ & 20 & & 62 & & 82 & \\
\hline Sindicatos de trabalhadores & 18 & 90,0 & 59 & 95,2 & 77 & 93,9 \\
\hline Conselho de Saúde & 14 & 70,0 & 58 & 93,5 & 72 & 87,8 \\
\hline Previdência Social & 12 & 60,0 & 34 & 54,8 & 46 & 56,1 \\
\hline Centrais sindicais & 17 & 85,0 & 28 & 45,2 & 45 & 54,9 \\
\hline Sindicatos ou representação patronal & 8 & 40,0 & 37 & 59,7 & 45 & 54,9 \\
\hline Associações ou conselhos profissionais & 11 & 55,0 & 32 & 51,6 & 43 & 52,4 \\
\hline Academia/Universidade & 13 & 65,0 & 24 & 38,7 & 37 & 45,1 \\
\hline Ministério do Trabalho/SRTE & 10 & 50,0 & 16 & 25,8 & 26 & 31,7 \\
\hline Vigilância Epidemiológica & 2 & 10,0 & 21 & 33,9 & 23 & 28,0 \\
\hline Vigilância Ambiental & 3 & 15,0 & 16 & 25,8 & 19 & 23,2 \\
\hline Ministério Público & 4 & 20,0 & 11 & 17,7 & 15 & 18,3 \\
\hline Incra & 2 & 10,0 & 1 & 1,6 & 3 & 3,7 \\
\hline Outros & 11 & 55,0 & 26 & 41,9 & 37 & 45,1 \\
\hline \multicolumn{7}{|l|}{ Participação de trabalhadores } \\
\hline Na programação anual de 2008 ( $n=127)$ & 16 & 66,7 & 86 & 83,5 & 102 & 80,3 \\
\hline \multicolumn{7}{|c|}{$\begin{array}{l}\text { Nas reuniões de planejamento e avaliação das ações } \\
\text { do Cerest }(n=125)\end{array}$} \\
\hline Nunca & 6 & 24,0 & 12 & 12,0 & 18 & 14,4 \\
\hline Raramente & 5 & 20,0 & 15 & 15,0 & 20 & 16,0 \\
\hline Às vezes & 6 & 24,0 & 17 & 17,0 & 23 & 18,4 \\
\hline Frequentemente & 5 & 20,0 & 34 & 34,0 & 39 & 31,2 \\
\hline Sempre & 3 & 12,0 & 22 & 22,0 & 25 & 20,0 \\
\hline \multicolumn{7}{|l|}{$\begin{array}{l}\text { Nas ações de Visat }{ }^{* * * * * * * *} \text { em ambientes de trabalho } \\
(n=125)\end{array}$} \\
\hline Nunca & 6 & 25,0 & 18 & 17,8 & 24 & 19,2 \\
\hline Raramente & 6 & 25,0 & 26 & 25,7 & 32 & 25,6 \\
\hline Às vezes & 4 & 16,7 & 35 & 34,7 & 39 & 31,2 \\
\hline Frequentemente & 6 & 25,0 & 19 & 18,8 & 25 & 20,0 \\
\hline Sempre & 2 & 8,3 & 3 & 3,0 & 5 & 4,0 \\
\hline
\end{tabular}

* Os dados "n" se referem ao número de Cerests respondentes

***: Os dados das colunas № se referem ao número de respostas dos Cerests em cada quesito.

粎: Cist - Comissões Intersetoriais de Saúde do Trabalhador

粎䊎 Visat - Vigilância em Saúde do Trabalhador

Fonte: $1^{\circ}$ Inventário de Saúde do Trabalhador: Avaliação da Rede Nacional de Atenção Integral em Saúde do Trabalhador, 2008-2009 (BRASIL, 2010, p. 28) 
Tabela 4 Distribuição de implantação e avaliação das ações de Visat e articulações intra e intersetorial Brasil, 2009

\begin{tabular}{|c|c|c|c|c|c|c|}
\hline \multirow{2}{*}{ Variáveis } & \multicolumn{2}{|c|}{ Cerests estaduais } & \multicolumn{2}{|c|}{ Cerests regionais } & \multicolumn{2}{|c|}{ Total } \\
\hline & №* & $\%$ & $N^{\circ}$ & $\%$ & № & $\%$ \\
\hline \multicolumn{7}{|c|}{$\begin{array}{l}\text { Inspeções para avaliações de ambientes de trabalho } \\
(\mathrm{n}=125)^{* * *}\end{array}$} \\
\hline Não previstas & 2 & 9,1 & 3 & 2,9 & 5 & 4,0 \\
\hline Previstas & 1 & 4,5 & 8 & 7,8 & 9 & 7,2 \\
\hline Em fase de planejamento & 4 & 18,2 & 10 & 9,7 & 14 & 11,2 \\
\hline Em fase de implantação & 4 & 18,2 & 20 & 19,4 & 24 & 19,2 \\
\hline Em funcionamento & 8 & 36,4 & 44 & 42,7 & 52 & 41,6 \\
\hline Em funcionamento pleno & 3 & 13,6 & 18 & 17,5 & 21 & 16,8 \\
\hline \multicolumn{7}{|c|}{$\begin{array}{l}\text { Origem de demanda frequentemente atendida } \\
(n=143)^{* * *}\end{array}$} \\
\hline Sindicatos & 7 & 43,7 & 31 & 37,8 & 38 & 38,8 \\
\hline Ministério Público & 7 & 43,8 & 26 & 32,5 & 33 & 34,4 \\
\hline Comunidade & 0 & - & 16 & 20,5 & 16 & 17,4 \\
\hline Denúncia anônima & 1 & 7,1 & 13 & 16,5 & 14 & 15,1 \\
\hline Serviço sentinela & 4 & 26,7 & 28 & 35,4 & 32 & 34,0 \\
\hline Outros serviços da rede & 1 & 7,1 & 19 & 24,1 & 20 & 21,5 \\
\hline \multicolumn{7}{|c|}{$\begin{array}{l}\text { Avaliação das ações de vigilância de processos e ambi- } \\
\text { entes de trabalho }(n=123)^{* * *}\end{array}$} \\
\hline Não previstas & 2 & 9,5 & 8 & 7,8 & 10 & 8,1 \\
\hline Previstas & 9 & 42,9 & 30 & 29,4 & 39 & 31,7 \\
\hline Em fase de planejamento & 3 & 14,3 & 20 & 19,6 & 23 & 18,7 \\
\hline Em fase de implantação & 2 & 9,5 & 16 & 15,7 & 18 & 14,6 \\
\hline Em funcionamento & 5 & 23,8 & 28 & 27,5 & 33 & 26,8 \\
\hline Em funcionamento pleno & - & - & - & - & - & - \\
\hline
\end{tabular}


Na Tabela 4, pode-se observar que a origem da demanda atendida pelos Cerests provém principalmente dos sindicatos $(38,8 \%)$, seguida pelo MP $(34,4 \%)$ e por serviços sentinela $(34,0 \%)$, isto é, pela notificação. É maior a participação dos dois primeiros segmentos nos Cerests estaduais em comparação com os regionais, tendo os serviços sentinela maior participação relativa nos regionais. Isto ressalta a importância de representações da sociedade, já mencionada, e também a estruturação da rede sentinela. Esta é uma atividade fundamental para o sucesso das ações de Visat. No entanto, à época do registro dos dados analisados, os serviços sentinela estavam implantados em poucos Cerests. Apenas 45,5\% (10/22) dos Cerests estaduais e $28,7 \%$ dos regionais referiram rede sentinela "em funcionamento pleno" ou "em funcionamento". A região que apresentava melhor situação foi a Centro-Oeste, com todos os estados participantes referindo funcionamento pleno. Isso ocorreu em menor escala na região $\mathrm{Su}-$ deste (2/3) para os Cerests estaduais. Nos Cerests regionais, vale destacar que, na região Norte, apenas os Cerests do estado do Tocantins possuíam rede sentinela estruturada. Praticamente todos os Cerests regionais com rede sentinela em funcionamento estavam realizando capacitação dos profissionais destes serviços para atender às demandas em Saúde do Trabalhador, com exceção de dois $(n=2)$. Programas especiais estavam sendo realizados por $61,9 \%$ dos Cerests estaduais e por $47,1 \%$ dos regionais, sendo esta uma estratégia a ser incentivada e com grau de qualificação das ações de vigilância implantadas passível de acompanhamento.

Cerca de 1/3 de ambos os tipos de Cerest ainda se encontravam com ações de inspeção de ambientes de trabalho em fase de implantação, revelando a incipiência dessa importante atividade para a vigilância. Observa-se também que essa atividade era menos desenvolvida pelos Cerests estaduais, o que surpreende por se esperar destes maior grau de desenvolvimento e avanço dessas ações, aceitas como de mais difícil execução por serviços com menor tempo de implantação. Há que se considerar que boa parte da economia informal e dos trabalhadores informais não se encontra em espaços caracterizados tradicionalmente como ambientes de trabalho e que também devem ser alvo da atenção das inspeções do Cerest. Além disso, uma integração com a Vigilância Sanitária é esperada, valendo notar que, tradicionalmente, esta se volta mais para a segurança do consumidor do que propriamente dos trabalhadores.

A análise do processo de trabalho e da produção, contemplando cadeias produtivas, quando aplicável, seja documental ou por histórias e relatos dos tra- balhadores, sempre permite contribuições valiosas para a compreensão e a ação mais adequadas. Vale lembrar que, conforme já mencionado, qualquer que seja a fonte do conhecimento, a participação dos trabalhadores é fundamental (BRASIL, 1998).

Observou-se uma grande diversidade na origem das reivindicações por ações da Renast que foram atendidas. Sobressai a demanda por identificação e definição de nexo causal para casos de agravos suspeitos de relação com o trabalho. Esta é uma ação de apoio às lutas de reconhecimento dos direitos dos trabalhadores vinculados ao Instituto Nacional de Seguridade Social (INSS) e revela a falha do seu sistema médico pericial e, no geral, da identificação dos casos. Isso se evidencia no bem estudado problema de subnotificação e sub-registro de agravos relacionados ao trabalho. Assim, essa demanda traduz de modo emblemático que no SUS há um melhor acolhimento das lutas individuais dos trabalhadores diante dos processos institucionais de garantia de seus direitos. É uma ação importante, mas, vale ressaltar, distanciada dos objetivos de promoção da saúde e intervenção da Visat nos determinantes sanitários.

Em 2008, foram capacitados 16.457 profissionais de unidades sentinela por 66 Cerests que realizaram esta atividade, o que corresponde a um grande contingente e uma boa perspectiva de intensificação da vigilância epidemiológica dos agravos relacionados ao trabalho nas regiões relacionadas a esse grupo de serviços.

\section{Considerações finais}

Os resultados deste primeiro estudo de avaliação nacional da Renast mostram uma significativa adesão, nesse processo, por parte das equipes dos Cerests. Isto aponta para a factibilidade da institucionalização dessa avaliação, atendendo assim a um anseio da gestão do SUS, que é o de alcançar transparência das ações para a sociedade e o uso racional dos recursos. Dentre os Cerests não participantes, muitos poderiam estar, à época, em uma fase inicial de implantação e, assim, a sua participação é esperada para a próxima etapa de avaliação, contribuindo para o contínuo processo de aperfeiçoamento da Renast. Vale notar que esta é apenas uma parte inicial do processo, que deve culminar com a incorporação do conhecimento gerado no planejamento e na gestão.

Ao longo do texto foram apresentadas recomendações para as diferentes dimensões empregadas na avaliação. O Quadro 1 apresenta uma síntese das recomendações gerais com suas dimensões e seus objetivos, ações estratégicas e responsáveis por sua execução. 
Quadro 1 Recomendações gerais com suas dimensões e objetivos, ações estratégicas e responsáveis por sua execução

\begin{tabular}{|c|c|c|}
\hline Dimensões/objetivos & Ações estratégicas & Instituições responsáveis pela execução \\
\hline $\begin{array}{l}\text { Oferta de serviços de Saúde do Traba- } \\
\text { Ihador pelo SUS } \\
\text { Ampliar a cobertura dos Cerests } \\
\text { regionais }\end{array}$ & $\begin{array}{l}\text { Negociar a implantação de novos Cerests regio- } \\
\text { nais da região Norte, em especial Acre, Rondônia } \\
\text { e Amapá } \\
\text { Revisar a adequação da estrutura e o formato da } \\
\text { regionalização adotada na Renast }\end{array}$ & $\begin{array}{l}\text { Prefeituras municipais, secretarias } \\
\text { estaduais e Ministério da Saúde }\end{array}$ \\
\hline $\begin{array}{l}\text { Garantir a continuidade do finan- } \\
\text { ciamento e a eficiência e a agilidade } \\
\text { na execução orçamentária }\end{array}$ & $\begin{array}{l}\text { Elaborar planos de aplicação financeiros a serem } \\
\text { incluídos na Programação Anual de Saúde dos } \\
\text { Planos estaduais e municipais de saúde } \\
\text { Definir metas (quantitativas) para avaliação dos } \\
\text { resultados alcançados e das etapas implementadas } \\
\text { Analisar e induzir modos ágeis e eficientes de } \\
\text { repasse dos recursos e de sua gestão e utilização } \\
\text { pelo pessoal encarregado da execução local }\end{array}$ & $\begin{array}{l}\text { Secretarias estaduais e municipais de } \\
\text { saúde } \\
\text { Representantes dos trabalhadores e } \\
\text { dos movimentos sociais } \\
\text { Parceiros interinstitucionais }\end{array}$ \\
\hline $\begin{array}{l}\text { Melhoria das instalações físicas dos } \\
\text { Cerests }\end{array}$ & $\begin{array}{l}\text { Realizar visitas aos Cerests com problemas de } \\
\text { instalações físicas para o estabelecimento de } \\
\text { projetos de modo a captar recursos para sua } \\
\text { melhoria }\end{array}$ & $\begin{array}{l}\text { Ministério da Saúde, secretarias } \\
\text { estaduais de saúde }\end{array}$ \\
\hline $\begin{array}{l}\text { Adequar e qualificar as equipes do } \\
\text { Cerests }\end{array}$ & $\begin{array}{l}\text { Disseminar conhecimento dos fundamentos do } \\
\text { processo de vigilância em saúde do trabalhador }\end{array}$ & $\begin{array}{l}\text { Ministério da Saúde, secretarias } \\
\text { estaduais e municipais de saúde e ins- } \\
\text { tituiçõos acadêmicas (universidades, } \\
\text { Fundacentro e Fiocruz) }\end{array}$ \\
\hline & $\begin{array}{l}\text { Organizar Cist } \\
\text { Incluir trabalhadores nas ações de Visat }\end{array}$ & $\begin{array}{l}\text { Secretarias estaduais e municipais de } \\
\text { saúde }\end{array}$ \\
\hline $\begin{array}{l}\text { Ações desenvolvidas } \\
\text { Estruturar e fortalecer a rede } \\
\text { sentinela } \\
\text { Fortalecer a Visat }\end{array}$ & $\begin{array}{l}\text { Disseminar informações e conhecimento sobre } \\
\text { Visat } \\
\text { Estabelecimento de programas de Visat voltados } \\
\text { para problemas de saúde do trabalhador identifi- } \\
\text { cados a partir de análise de situação de saúde do } \\
\text { trabalhador, incluindo dados sobre processos } \\
\text { produtivos, riscos e impacto na saúde } \\
\text { Estabelecer dinâmica de discussão da importân- } \\
\text { cia e impacto da Visat }\end{array}$ & $\begin{array}{l}\text { Secretarias estaduais e municipais de } \\
\text { saúde } \\
\text { Representantes dos trabalhadores e } \\
\text { dos movimentos sociais } \\
\text { Parceiros interinstitucionais. } \\
\text { Ministério da Saúde }\end{array}$ \\
\hline $\begin{array}{l}\text { Reforçar os procedimentos de } \\
\text { vigilância epidemiológica } \\
\text { Disseminar a informação e estimu- } \\
\text { lar o debate para os atores sociais } \\
\text { de relevância para o tema }\end{array}$ & $\begin{array}{l}\text { Estabelecer informações de problemas de saúde } \\
\text { do trabalhador (processos, riscos e impacto) } \\
\text { Estabelecer um processo de planejamento } \\
\text { calcado na análise de situação de saúde }\end{array}$ & $\begin{array}{l}\text { Secretarias estaduais e municipais de } \\
\text { saúde } \\
\text { Representantes dos trabalhadores e } \\
\text { dos movimentos sociais } \\
\text { Parceiros interinstitucionais } \\
\text { Ministério da Saúde }\end{array}$ \\
\hline
\end{tabular}

Fonte: $1^{1}$ Inventário de Saúde do Trabalhador: Avaliação da Rede Nacional de Atenção Integral em Saúde do Trabalhador, 2008-2009 (BRASIL, 2010, p. 50) 
Frise-se que a gestão da Renast ainda se ressente de melhor estruturação e adequação às singularidades da sua proposta. Dentre os pontos principais, destacam-se a garantia da continuidade do financiamento e da contrapartida dos governos locais, a agilidade restrita na aplicação dos recursos e a falta do estabelecimento de prioridades com base em informações epidemiológicas e do perfil produtivo. Há uma clara lacuna na produção de informações sobre o impacto dos programas e investimentos na melhoria das condições de trabalho e saúde dos trabalhadores. Nota-se que a produção de informações deve ser um foco prioritário de fortalecimento institucional, que em conjunto com a participação dos trabalhadores nas ações qualificam o processo de vigilância.

Em síntese, a avaliação das ações de vigilância e informação em Saúde do Trabalhador possibilita a identificação de três grupos de Cerests com graus distintos de implantação. Um primeiro, que pode ser considerado com implantação adequada, um segundo, em processo de implantação e um terceiro, em fase inicial. Devem-se buscar formas de aproximação desses três grupos em um processo de integração em rede, mediado pela Renast, via contatos regionais e programas de qualificação dos profissionais para exercício da gestão e da melhoria da capacidade de resposta dos próprios serviços. É também essencial criar mecanismos de aproximação com outras instâncias das redes de atenção à saúde do SUS e estabelecer a interação com outros setores e com os movimentos sociais. Nesse sentido, a consolidação de um processo de avaliação pode ser favorável à construção de um cenário da rede, com um entendimento da dimensão estrutural, das equipes e do custeio dos Cerests.

Esse processo de avaliação deve ser aprofundado e integrado com o SUS em todas as unidades federadas e deve ser complementado com outras abordagens, buscando um aperfeiçoamento contínuo da Renast. A qualidade das respostas e do processo de acompanhamento da Renast deve ser também objeto de constante aperfeiçoamento. A próxima consulta para avaliação utilizará um novo instrumento, que permite maior agilidade nos resultados, assegurando sua utilização para subsidiar mudanças favoráveis aos avanços no compromisso com a Saúde do Trabalhador.

Alguns pressupostos devem ser enfatizados nesse processo de aperfeiçoamento:

- A participação dos trabalhadores nas ações de Visat devido à sua característica essencial ao modelo de vigilância adotado de acordo com Portaria $n^{\circ}$ 3.252/09 (BRASIL, 2009) e as Dire- trizes das ações de Vigilância em Saúde do Trabalhador (BRASIL, 1998; 2012).

- As ações de vigilância em saúde do trabalhador organizadas a partir dos Cerests com avaliação contínua e realizadas de forma sistemática.

O SUS e o seu modelo de atenção à saúde do trabalhador, de caráter universal e incorporada à atenção primária de saúde, poderão, potencialmente, atingir todos os trabalhadores brasileiros. Esta integração com a atenção básica, o aperfeiçoamento da vigilância e a produção de informações precisas e abrangentes são alguns dos seus principais desafios.

Uma síntese das informações, com a sugestão de metas a serem avaliadas em 2012, são apresentadas na Tabela 5. Esses parâmetros e variáveis de acompanhamento são patamares que podem ser pactuados com as instâncias coordenadoras dos estados e municípios.

Finalmente, no sentido de contribuição para uma avaliação sistemática das ações de Visat propõe-se o acompanhamento de pelo menos quatro dimensões desta ação:

1) O registro das informações, com o fortalecimento da participação dos Cerests na formação da rede sentinela de notificação de agravos relacionados ao trabalho, bem como na utilização de fontes como oSistema de Informação sobre a Mortalidade (SIM), o Sistema de Notificação dos Agravos Notificáveis (Sinan) e o Sistema de Informações da Atenção Básica (Siab), além da articulação direta com outras redes assistenciais, como os Centros de Atenção Psicossocial (CAPs) e de atenção básica.

2) A análise das informações a partir das múltiplas demandas assistenciais e de vigilância, com a produção de perfis epidemiológicos e de situações de risco e vulnerabilidades. Essa articulação da informação possibilita análises mais elaboradas utilizando-se dados demográficos e do perfil produtivo das regiões de referência dos serviços no sentido de estabelecer uma racionalidade epidemiológica na priorização das respostas dos Cerests.

3) A execução de ações de investigação de campo, com destaque para a execução de inspeções em ambientes de trabalho e para a organização de processos participativos e intersetoriais nas atividades de Visat.

4) A organização de ações programáticas, com o desenvolvimento de programas especiais para atender às demandas específicas e prioritárias dos trabalhadores da região de referência dos Cerests. 
Tabela 5 Síntese da avaliação dos Cerests estaduais e regionais segundo variáveis de estrutura e controle social, de ações desenvolvidas e de produção de informação (2008-2009), com suas respectivas metas para 2011-2012

\begin{tabular}{|c|c|c|c|c|c|}
\hline Variável & $\begin{array}{l}\text { Estadual } \\
\qquad \%)\end{array}$ & $\begin{array}{l}\text { Regional } \\
\text { (\%) }\end{array}$ & $\begin{array}{c}\text { Total } \\
(\%)\end{array}$ & $\begin{array}{c}\text { Metas } \\
2011-2012 \\
(\%)\end{array}$ & $\begin{array}{c}\text { Diferença } \\
\text { entre } \\
\text { realizado e metas } \\
\text { (\%) }\end{array}$ \\
\hline \multicolumn{6}{|l|}{ Estrutura e controle social } \\
\hline Equipe adequada & 50,0 & 59,0 & 57,4 & 70 & 12,6 \\
\hline Presença de Cist & 76,9 & 60,8 & 66,3 & 80 & 13,7 \\
\hline \multicolumn{6}{|l|}{ Ações desenvolvidas de Visat } \\
\hline Participação dos trabalhadores nas ações & 33,3 & 21,8 & 24,0 & 50 & 26,0 \\
\hline $\begin{array}{l}\text { Realização de inspeções nos ambientes } \\
\text { de trabalho }\end{array}$ & 50,0 & 60,2 & 58,4 & 70 & 11,6 \\
\hline Avaliação da Visat & 23,8 & 27,5 & 26,8 & 50 & 23,2 \\
\hline \multicolumn{6}{|c|}{ Ações desenvolvidas de Vigilância Epidemiológica } \\
\hline Acidente de trabalho fatal & 55,6 & 67,0 & 64,8 & 80 & 15,2 \\
\hline Acidente de trabalho infantil & 36,3 & 49,0 & 46,8 & 70 & 23,2 \\
\hline Pneumopatias & 28,5 & 39,6 & 36,9 & 50 & 13,1 \\
\hline Intoxicações & 45,5 & 53,8 & 52,4 & 70 & 17,6 \\
\hline LER/DORT & 54,6 & 59,2 & 58,4 & 70 & 11,6 \\
\hline Transtornos mentais & 36,4 & 34,9 & 35,2 & 50 & 14,8 \\
\hline \multicolumn{6}{|l|}{ Produção de informação } \\
\hline Informações demográficas & 33,3 & 15,8 & 18,8 & 50 & 31,2 \\
\hline Informações produção & 38,1 & 27,7 & 29,5 & 50 & 20,5 \\
\hline Análise mortalidade & 28,6 & 21,4 & 22,6 & 50 & 27,4 \\
\hline Análise morbidade & 14,3 & 12,6 & 12,9 & 50 & 37,1 \\
\hline
\end{tabular}

Fonte: $1^{0}$ Inventário de Saúde do Trabalhador: Avaliação da Rede Nacional de Atenção Integral em Saúde do Trabalhador, 2008-2009 (BRASIL, 2010, p. 51)

\section{Contribuições de autoria}

Machado, J. M. H.; Santana, V. S.: coordenaram a organização do inventário que originou o artigo e participaram do desenvolvimento da metodologia de avaliação adotada. Campos, A.: desenvolveu a aplicação de tecnologia da informação utilizada no inventário. Ferrite, S.: participou do desenvolvimento da base da metodologia de avaliação adotada. Peres, M. C.; Galdino, A.; Vasconcelos Neto, R.; Veiga, R. M. P.; Lisboa, M. C.; Santos, A. P. L.: colaboraram na análise e na apresentação de dados. Todos os autores participaram do desenvolvimento do trabalho e da elaboração ou revisão crítica do manuscrito.

\section{Referências}

BRASIL. Ministério da Saúde. Coordenação Geral de Saúde do Trabalhador. Renast Online. Diretrizes de implantação da vigilância em saúde do trabalhador no SUS, 2012. Disponível em: < http://www.renastonline. org/recursos/diretrizes-implanta\%C3\%A7\%С3\%А3оvigil\%C3\%A2ncia-sa\%C3\%BAde-trabalhador-sus $>$. Acesso em: 12 nov. 2013.
. Ministério da Saúde. Fundação Oswaldo Cruz. Universidade Federal da Bahia. $1^{\circ}$ Inventário de saúde do trabalhador, 2009: avaliação da rede nacional de atenção integral em saúde do trabalhador, 2008-2009. [S.I.]: Ministério da Saúde, Fiocruz, UFBA, 2011. Disponível em: <http://portal.saude.gov.br/portal/arquivos/pdf/ inventario_renast.pdf $>$. Acesso em: 12 nov. 2013. 
BRASIL. Ministério da Saúde. Portaria GM/MS no 3.120, de 01 de julho de 1998. Aprova a Instrução Normativa de Vigilância em Saúde do Trabalhador no SUS. Diário Oficial [da] República Federativa do Brasil, Poder Executivo, Brasília, DF, 2 jul. 1998. Disponível em: < http://www.cerest.piracicaba.sp. gov.br/site/images/3120_-_98.pdf $>$. Acesso em: 12 nov. 2013

. Ministério da Saúde. Portaria GM/MS nº 3.252 , de 22 de dezembro de 2009. Aprova as diretrizes para execução e financiamento das ações de Vigilância em Saúde pela União, Estados, Distrito Federal e Municípios e dá outras providências. Diário Oficial [da] República Federativa do Brasil, Poder Executivo. Brasília, DF, 23 dez. 2009. Disponível em: < http:// portal.saude.gov.br/portal/arquivos/pdf/portaria3252_ da_vigilancia_em_saude_0501_atual.pdf $>$. Acesso em 12 nov. 2013.

. Ministério da Saúde. Rede Nacional de Atenção Integral à Saúde do Trabalhador: manual de gestão e gerenciamento. São Paulo: Hemeroteca Sindical Brasileira, 2006. Disponível em: <http://portal.saude.gov.br/portal/arquivos/pdf/ ManualRenast07.pdf>. Acesso em: 12 nov. 2013.

DIAS, E. C.; HOEFEL, M. G. O desafio de implementar as ações de saúde do trabalhador no SUS: a estratégia da Renast. Ciência e Saúde Coletiva, Rio de Janeiro, v. 10, n. 4, p. 817-827, dez. 2005.

DIAS, E. C. et al. Construção da Renast em Minas Gerais: a contribuição dos Centros de Referência em Saúde do Trabalhador (Cerest), 2002-2007. Revista Médica de Minas Gerais, Belo Horizonte, v. 20, n. 2, supl. 2, p. 66-74, abr.-jun. 2010.

FACCHINI, L. A. et al. Desempenho do PSF no sul e no Nordeste do Brasil: avaliação institucional e epidemiológica da atenção Básica à Saúde. Ciência e Saúde Coletiva, Rio de Janeiro, v. 11, n. 3, p. 669-681, set. 2006.

GALDINO, A.; SANTANA, V. S.; FERRITE, S. Os Centros de Referência em Saúde do Trabalhador e a notificação de acidentes de trabalho no Brasil. Cadernos de Saúde Pública, Rio de Janeiro, v. 28, n. 1, p. 145-159, jan. 2012.

LACAZ, F. A. C.; MACHADO, J. M. H.; PORTO, M. F. S. Estudo da situação e tendências da vigilância em saúde do trabalhador no Brasil. Relatório final do projeto. Rio de Janeiro: OPAS, Abrasco, 2002. Disponível em: < http:/ www.abrasco.org.br/grupos/arquivos/20060717150425. pdf> . Acesso em: 13 nov. 2013.

MACHADO, J. M. H. Alternativas e processos de Vigilância em Saúde do Trabalhador: a heterogeneidade da intervenção. 1996. 166 f. Tese (Doutorado em Saúde Pública)-Escola Nacional de Saúde Pública, Fiocruz, Rio de Janeiro, 1996.

MACHADO, J. M. H.; SANTOS, A. P. L. O

financiamento na indução das ações de saúde do trabalhador no Brasil: o caso da Renast. In: MELAMED, C.; PIOLA, S. F (Org.). Políticas públicas e financiamento federal do Sistema Único de Saúde. Brasília: Ipea, 2011. p. 151-164.

RAMOS, J. C. L. Especialização em saúde do trabalhador no Brasil: estudo dos cursos no período 1986 - 2006. 2008. 81 f. Dissertação (Mestrado em Saúde Coletiva)-Instituto de Saúde Coletiva, Universidade Federal da Bahia, Salvador, 2008.

SANTANA, V. S.; SILVA, J. M. Os 20 anos da saúde do trabalhador no Sistema Único de Saúde do Brasil: limites, avanços e desafios. In: BRASIL. Ministério da Saúde. Secretaria de Vigilância em Saúde. Departamento de Análise de Situação em Saúde. Saúde Brasil 2008: 20 anos de Sistema Único de Saúde (SUS) no Brasil. Brasília: Ministério da Saúde, 2009. p. 175-204.

SANTOS, A. P. L. Tecendo redes: superando desafios, estudos de casos exitosos em saúde do trabalhador no Sistema Único de Saúde do Estado de São Paulo. 2010. 250 f. Tese (Doutorado em Ciências)-Departamento de Medicina Preventiva, Universidade Federal de São Paulo, São Paulo, 2010. 